\title{
Noninvasive Measurement of Inspiratory Muscle Performance by means of Diaphragm Muscle Mechanomyographic Signals in COPD patients during an Incremental Load Respiratory Test
}

\author{
Abel Torres, Member, IEEE, Leonardo Sarlabous, José A. Fiz, \\ Joaquim Gea, Juana M. Martínez-Llorens, J. Morera and Raimon Jané, Member, IEEE
}

\begin{abstract}
The study of mechanomyographic (MMG) signals of respiratory muscles is a promising noninvasive technique in order to evaluate the respiratory muscular effort and efficiency. In this work, the MMG signal of the diaphragm muscle it is evaluated in order to assess the respiratory muscular function in Chronic Obstructive Pulmonary Disease (COPD) patients. The MMG signals from left and right hemidiaphragm were acquired using two capacitive accelerometers placed on both left and right sides of the costal wall surface. The MMG signals and the inspiratory pressure signal were acquired while the COPD patients carried out an inspiratory load respiratory test. The population of study is composed of a group of 6 patients with severe COPD (FEV1 $>50 \%$ ref and DLCO $<50 \%$ ref). We have found high positive correlation coefficients between the maximum inspiratory pressure (IPmax) developed in a respiratory cycle and different amplitude parameters of both left and right MMG signals (RMS, left: $0.68 \pm 0.11$ - right: $0.69 \pm 0.12$; Rényi entropy, left: $0.73 \pm 0.10$ - right: $0.77 \pm 0.08$; Multistate LempelZiv, left: $0.73 \pm 0.17$ - right: $\mathbf{0 . 7 4} \pm \mathbf{0 . 0 8}$ ), and negative correlation between the Pmax and the maximum frequency of the MMG signal spectrum (left: $\mathbf{- 0 . 3 9} \pm 0.19$ - right: $\mathbf{- 0 . 6 5} \pm 0.09$ ). Furthermore, we found that the slope of the evolution of the MMG amplitude parameters, as the load increases during the respiratory test, has positive correlation with the \%FEV1/FVC pulmonary function test parameter of the six COPD patients analyzed (RMS, left: 0.38 - right: 0.41 ; Rényi entropy, left: 0.45 - right: 0.63; Multistate Lempel-Ziv, left: 0.39 - right: 0.64). These results suggest that the information provided by MMG signals could be used in order to evaluate the respiratory effort and the muscular efficiency in COPD patients.
\end{abstract}

This work was supported in part by grants from Ministerio de Educación y Ciencia of Spain (TEC2007-68076-C02-01) and in part by the Ministerio de Asuntos Exteriores y de Cooperación (AECID scholarship)

L. Sarlabous, A. Torres and R. Jané are with Dept. ESAII, Universitat Politècnica de Catalunya, Institut de Bioenginyeria de Catalunya (IBEC) and CIBER de Bioingeniería, Biomateriales y Nanomedicina (CIBERBBN), Barcelona, Spain (e-mail: leonardo.sarlabous@upc.edu, abel.torres@upc.edu, raimon.jane@upc.edu).

J. A. Fiz is with the Department of Pneumologie of the Navarra Hospital, Pamplona, Spain (e-mail: jfizfern@navarra.es).

J. Gea and J. M. Martínez-Llorens are with the Department of Respiratory Medicine at the IMIM- Hospital del Mar, UPF, CIBERES, Barcelona, Spain (e-mail: jgea@imim.es,jmartinezl@imas.imim.es).

J. Morera is with the Department of Pneumology of the Germans Trias i Pujol Hospital, Badalona, Spain (e-mail: josepmorera.germanstrias@gencat.net).

\section{INTRODUCTION}

$T$ HE surface mechanomyogram (MMG) is a noninvasive technique that quantifies the low-frequency lateral oscillations of the muscle fibers during contraction. These oscillations have been suggested to be a function of the gross lateral movement of the muscle and smaller lateral oscillations due to the summation of the muscle fibers movement from the recruited muscle motor units [1]. The time and frequency domain of the MMG signal has been used to study various aspects of muscle function. In general, a positive correlation coefficient has been found between amplitude parameters of the MMG signal and the force produced by the muscle [1]-[7].

The respiratory muscular activity and fatigue can be assessed by means of the implementation of techniques that study the muscular activity in amplitude and frequency through the MMG signal of respiratory muscles. The diaphragm is the main respiratory muscle responsible for the mechano-respiratory activity. In previous works [9]-[14], our group has analyzed, in an animal model (dogs), the signal acquired by means of a capacitive accelerometer placed on the costal wall of the thoracic cage in order to register the mechanomyographic (MMG) signal of the diaphragm muscle. In these works, a positive correlation has been found between amplitude parameters of the diaphragmatic MMG signal and the respiratory effort assessed by means of the inspiratory pressure.

In [15] these results were extrapolated to human experiments in a study of diaphragmatic MMG signals acquired during a non fatiguing incremental flow respiratory test in a population of severe Chronic Obstructive Pulmonary Disease (COPD) patients. In patients COPD the respiratory muscular function and the mechanic of the thoracic cage are very affected. The muscular efficiency is very reduced, due to the changes in the thoracodiaphragmatic space configuration produced by the disease, and that makes ineffective the muscular contraction, wasting great amounts of energy.

The aim of the present work is to evaluate the performance of the diaphragmatic MMG amplitude and frequency parameters acquired during a fatiguing incremental load respiratory test in order to assess the respiratory muscular function in COPD patients. 


\section{MEthodOLOGY}

\section{A. Study population}

The study population consisted of six patients with severe COPD, with smoking antecedents and diagnosed by means of forced spirometry and TAC illustrative of lung emphysema. Table I reports the clinical characteristics and the parameters of the pulmonary function tests of this group of COPD patients.

\section{B. Signals and instrumentation}

The subjects were instrumented in order to acquire three biomedical signals: the inspiratory pressure (IP) and the mechanomyographic signals of the left and right hemidiaphragms (MMGl and MMGr, respectively). IP was measured with a pressure transducer placed in the tube through which the patients breathe. MMGl and MMGr were acquired with two Kistler 8312B2 capacitive accelerometers placed on the surface of the thoracic cage. The placement of the sensors (between the seventh and eighth intercostal spaces in the left and right anterior axillary lines) was chosen with the intention of obtaining the mechanomyographic signal of both left and right hemidiaphragms.

All analog signals were amplified, analog filtered, digitized with a 12 bit A/D system at a sampling rate of 4 $\mathrm{kHz}$, and decimated at a new sampling rate of $200 \mathrm{~Hz}$.

\section{Respiratory protocol}

The six COPD patients performed an incremental inspiratory load protocol. During the respiratory protocol the subject is seated and breathes through the mouth by means of a mouthpiece and a tube, and with a nose clip.

During the exhalation the tube allows the air outlet without obstruction. However in the inspiration the tube is blocked out by a valve which allows us to hang small weights. To inhale air the subject must perform an inspiratory pressure to open up the valve by lifting the weights. The test begins with quiet respiration at basal tidal volume (without any load), and every two minutes it is added a load of 50 grams (equivalent to a generation of 10 $\mathrm{cmH} 2 \mathrm{O}$ of inspiratory pressure, approximately). The test
TABLE II

DURATION, NUMBER OF CYCLES AND MAXIMUM INSPIRATORY PRESSURE DEVELOPED IN THE RESPIRATORY TESTS

\begin{tabular}{ccccc}
\hline \hline Patient & $\begin{array}{c}\text { Duration } \\
(\mathrm{s})\end{array}$ & $\begin{array}{c}\text { Number of } \\
\text { cycles }\end{array}$ & Load (g) & $\begin{array}{c}\text { Maximum } \\
\text { IP } \\
(\mathrm{cmH} 2 \mathrm{O})\end{array}$ \\
\hline COPD1a & 387 & 127 & 100 & 33.4 \\
COPD1b & 457 & 143 & 100 & 34.7 \\
COPD2a & 677 & 211 & 200 & 54.4 \\
COPD2b & 749 & 262 & 250 & 63.6 \\
COPD3a & 459 & 191 & 100 & 35.3 \\
COPD3b & 622 & 216 & 200 & 56.2 \\
COPD4a & 648 & 242 & 200 & 55.6 \\
COPD4b & 623 & 256 & 200 & 50.2 \\
COPD5a & 384 & 110 & 100 & 30.3 \\
COPD5b & 383 & 122 & 100 & 31.8 \\
COPD6a & 290 & 112 & 50 & 24.2 \\
$-\mathrm{Me} \pm$ & $516.3 \pm$ & $181.1 \pm$ & $145.5 \pm$ & $42.7 \pm$ \\
STD & 151.6 & 59.8 & 65.0 & 13.4 \\
\hline \hline
\end{tabular}

ends when the subject can not hold the inspiratory load. In this way, during the respiratory test there is an intense breathing pattern and at the end of the test respiratory muscle fatigue is reached. During the realization of the respiratory tests, the medical personnel instruct to the patients in order to perform correctly the protocol and to maintain constant the rhythm and intensity of the breathing activity.

The increment of the respiratory load causes an increase of the inspiratory pressure and of the respiratory muscular effort. This increase in the respiratory muscular effort can be analyzed by means of the MMG signals.

Fig. 1 shows an example of the IP, MMGl and MMGr signals corresponding to an incremental inspiratory load protocol. As it can be seen, during the test the respiratory effort (assessed by means the inspiratory pressure) increases approximately $10 \mathrm{cmH} 2 \mathrm{O}$ every 2 minutes.

The duration, number of respiratory cycles, weight supported and maximum inspiratory pressure reached during the inspiratory load test by each patient are shown in Table II. The inspiratory load protocol was repeated two times for each patient, except for the sixth patient (COPD6).

TABLE I

PATIENTS CHARACTERISTICS AND PARAMETERS OF THE PULMONARY FunCTION TESTS

\begin{tabular}{ccccccccc}
\hline \hline & Age (years) & Height $(\mathrm{cm})$ & Weight $(\mathrm{kg})$ & FEV $_{1} \%$ ref. & FVC \% ref. & \%FEV FVC $_{1}$ & DLco \% ref. & Kco \% ref. \\
\hline COPD1 & 51 & 166 & 53 & 18 & 49 & 28.63 & 18 & 39 \\
COPD2 & 65 & 162 & 58 & 24 & 42 & 41.17 & 38 & 60 \\
COPD3 & 75 & 167 & 72 & 36 & 52 & 48.62 & 42 \\
COPD4 & 74 & 164 & 45 & 35 & 56 & 43.66 & 44 & 56 \\
COPD5 & 67 & 173 & 77 & 22 & 43 & 34.63 & 42 & 54 \\
COPD6 & 77 & 166 & 79 & 44 & 62 & 49.41 & 63 \\
Me \pm STD & $68.2 \pm 9.6$ & $166.3 \pm 3.7$ & $64.0 \pm 14.0$ & $29.8 \pm 10.0$ & $50.7 \pm 7.7$ & $41.0 \pm 8.1$ & $41.2 \pm 14.4$ & $57.3 \pm 10.7$ \\
\hline \hline
\end{tabular}

$\mathrm{FEV}_{1}=$ forced expiratory volume over one second, $\mathrm{FVC}=$ forced vital capacity, $\mathrm{FEV}_{1} / \mathrm{FVC}=$ proportion of the forced vital capacity exhaled in the first second, $\mathrm{DLco}=$ carbon monoxide diffusing capacity, $\mathrm{Kco}=$ carbon monoxide transfer coefficient $\%$ ref $=$ percentage regarding the predicted value, $\mathrm{Me}=$ mean, $\mathrm{STD}=$ standard deviation 

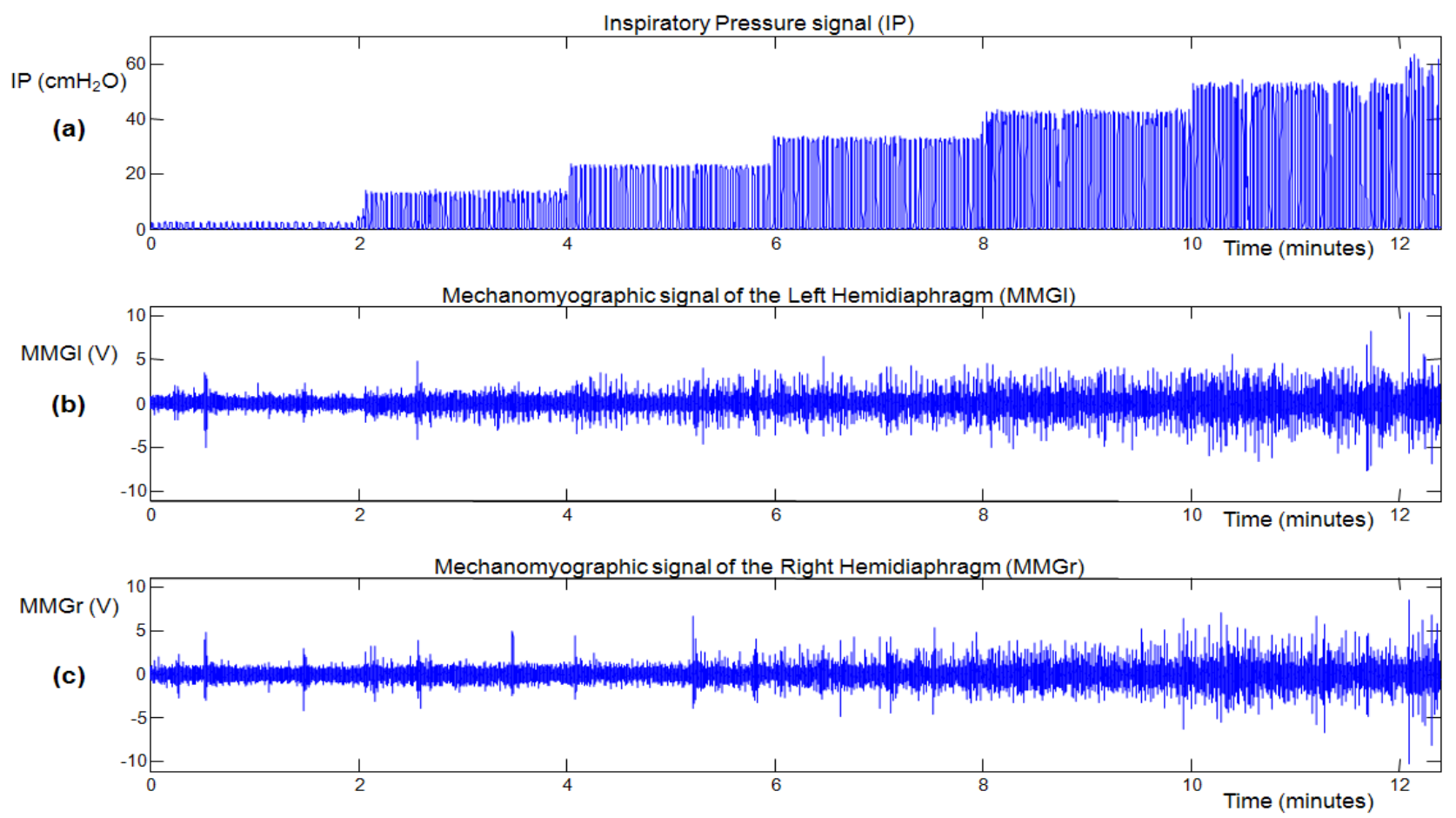

Fig. 1. Example of signals acquired during the incremental inspiratory load respiratory test: (a) Inspiratory pressure (IP) signal, (b) left diaphragm mechanomyographic (MMGl) signal, (c) right diaphragm mechanomyographic (MMGr) signal

\section{Signal Processing}

Identification of respiratory cycles and detection of initial and final time of diaphragm muscle contraction was made by means of the IP signal. In each respiratory cycle the maximum inspiratory pressure $\left(\mathrm{IP}_{\max }\right)$ achieved was determined. In order to quantify the changes in amplitude and frequency in the MMG signals, four parameters were estimated in both MMGl and MMGr signals:

- the root mean square (RMS)

- the Rényi entropy with $\alpha=0.5(\mathrm{H})$

- the Multistate Lempel-Ziv coefficient (LZM)

- the Maximum Frequency (fmax)
The explanation of the Rényi entropy and MLZ estimation techniques are detailed in [14] and [15], respectively.

The relationship between the IP parameter $\left(\mathrm{IP}_{\max }\right)$ and MMG parameters (RMS, H, LZ and fmax) were analyzed by means of the Pearson correlation coefficient.

\section{RESULTS}

Correlation coefficients between the maximum inspiratory pressure developed $\left(\mathrm{IP}_{\max }\right)$ and the diaphragmatic $\mathrm{MMG}$ parameters (RMS, H, MLZ and fmax) for the six COPD patients analyzed are shown in Table III.

TABLE III

CORRELATION COEFFICIENTS BETWEEN INSPIRATORY PRESSURE AMPLITUDE AND THE MMG PARAMETERS

\begin{tabular}{|c|c|c|c|c|c|c|c|c|}
\hline & \multicolumn{4}{|c|}{ Left MMG } & \multicolumn{4}{|c|}{ Right MMG } \\
\hline & RMS & $\mathrm{H}$ & MLZ & fmax & RMS & $\mathrm{H}$ & MLZ & fmax \\
\hline COPD1a & 0.699 & 0.760 & 0.760 & -0.599 & 0.794 & 0.831 & 0.760 & -0.534 \\
\hline COPD2a & 0.738 & 0.823 & 0.934 & -0.310 & 0.563 & 0.671 & 0.605 & -0.707 \\
\hline COPD2b & 0.852 & 0.870 & 0.854 & -0.268 & 0.767 & 0.833 & 0.800 & -0.660 \\
\hline COPD4a & 0.595 & 0.692 & 0.680 & -0.528 & 0.454 & 0.604 & 0.613 & -0.547 \\
\hline COPD4b & 0.709 & 0.778 & 0.783 & -0.403 & 0.662 & 0.761 & 0.754 & -0.552 \\
\hline COPD5a & 0.660 & 0.680 & 0.527 & -0.586 & 0.777 & 0.816 & 0.801 & -0.626 \\
\hline COPD5b & 0.466 & 0.542 & 0.353 & 0 & 0.651 & 0.714 & 0.693 & -0.652 \\
\hline
\end{tabular}


TABLE IV

CORRELATION COEFFICIENTS BETWEEN THE SLOPE VARIATION OF THE MMG PARAMETERS AND THE PULMONARy FunCtiON TESTS PARAMETERS

\begin{tabular}{|c|c|c|c|c|c|c|c|c|}
\hline & \multicolumn{4}{|c|}{ Left MMG } & \multicolumn{4}{|c|}{ Right MMG } \\
\hline & RMS & $\mathrm{H}$ & MLZ & fmax & RMS & $\mathrm{H}$ & MLZ & fmax \\
\hline $\mathrm{FEV}_{1} \%$ ref. & 0.16 & 0.33 & 0.24 & -0.02 & 0.05 & 0.42 & 0.42 & -0.34 \\
\hline FVC \% ref. & -0.17 & 0.13 & 0.03 & -0.32 & -0.39 & 0.14 & 0.14 & 0.15 \\
\hline$\% \mathrm{FEV}_{1} / \mathrm{FVC}$ & 0.38 & 0.45 & 0.39 & 0.07 & 0.41 & 0.63 & 0.64 & -0.67 \\
\hline DLco \% ref. & 0.08 & 0.08 & 0.04 & 0.49 & 0.05 & 0.12 & 0.10 & -0.33 \\
\hline Kco \% ref. & -0.09 & -0.10 & -0.19 & 0.43 & 0.09 & 0.19 & 0.23 & -0.49 \\
\hline
\end{tabular}

RMS = Root Mean Square, $\mathrm{H}=$ Rényi entropy, MLZ = Multistate Lempel-Ziv, fmax = Maximum frequency, $\mathrm{FEV}_{1}=$ forced expiratory volume over one second, $\mathrm{FVC}=$ forced vital capacity, $\mathrm{FEV}_{1} / \mathrm{FVC}=$ proportion of the forced vital capacity exhaled in the first second, DLco = carbon monoxide diffusing capacity, $\mathrm{Kco}=$ carbon monoxide transfer coefficient $\% \mathrm{ref}=$ percentage regarding the predicted value, $\mathrm{Me}=$ mean, $\mathrm{STD}=$ standard deviation.

Positive high correlation coefficients were found for MMG amplitude parameters, whereas negative correlation coefficients were found for MMG maximum frequency. That means that, when the IP increases, an augment of amplitude and a displacement towards lower frequencies takes place in the MMG signals. This behavior is observed in a similar way in both hemidiaphragms (MMGl and MMGr) and coincides with the results obtained in a previous study [15], in which it was analyzed the behavior of these signals during the implementation of an incremental flow respiratory protocol (without inspiratory loads).

Also as in [15], in the comparison of the performance of the three analyzed MMG amplitude parameters (RMS, H and MLZ), the entropy parameter $(\mathrm{H})$ is the one with better average, and the behavior of the RMS parameter in all the tests is inferior to the behavior of the $\mathrm{H}$ parameter, as concluded in [13] in an study of diaphragmatic MMG signals acquired in an animal model (dogs).

Table IV shows the correlation coefficients between the slope of the evolution of MMG parameters with the increment of IP, and the pulmonary function test parameters (FEV $\mathrm{FE}_{1} \%$ ref., $\mathrm{FVC} \%$ ref., $\% \mathrm{FEV}_{1} / \mathrm{FVC}, \mathrm{DLco} \%$ ref., and $\mathrm{Kco} \%$ ref.) for the six COPD patients.

\section{CONCLUSIONS}

In this work diaphragmatic MMG signals acquired during an incremental inspiratory load respiratory protocol in a COPD patient population have been analyzed. It has been observed that as in [15] the increments of inspiratory pressure that take place during the test are correlated with increases of amplitude and displacements toward low frequencies in the respiratory muscles MMG signals.

However, unlike in [15], in this study it was not found that the behavior of the slope of the variations in amplitude and frequency with pressure depends on the severity of the COPD patient. Only in the $\% \mathrm{FEV}_{1} / \mathrm{FVC}$ parameter it was slightly found that the slope of the increment in amplitude was lower in severe COPD patients.

The results obtained in this work suggest that the information provided by MMG signals could be used in order to evaluate the respiratory effort and the muscular efficiency in COPD patients.

[1] C. Orizio, "Muscle Sound: bases for the introduction of a mechanomyogaphic signal in muscle studies," Crit. Rev. Biomed. Eng., 21, pp. 201-243, 1993

[2] M. Petitjean, B. Maton, and J.-C. Cnockaert, "Evaluation of human dynamic contraction by phonomyography", J. Appl. Physiol., 73, pp. 2567-2573, 1992

[3] D. B. Smith, T. J. Housh, G. O. Johnson, T. K. Evetovixh, K. T. Ebersole, and S. R. Perry, "Mechanomyographic and electromyographic responses to eccentric and concentric isokinetic muscle actions of the biceps brachii", Muscle \& Nerve, 21, pp. 14381444,1998

[4] J. Celichowski, K. Grottel, and E. Bichler, "Relationship between mechanomyogram signals and changes in force of human forefinger flexor muscles during voluntary contraction", Eur. J. Appl. Physiol., 78, pp. 283-288, 1998

[5] M. J. Stokes and P. A. Dalton, "Acoustic myographic activity increases linearly up to maximal voluntary isometric force in the human quadriceps muscle," J. Neurol. Sci., 101, pp.163-167, 1991.

[6] F. Esposito, D. Malgrati, A. Veicsteinas and C. Orizio, "Time and frequency domain analysis of electromyogram and soundmyogram in the elderly,” Eur. J. Appl. Physiol., 73, pp.503-510, 1996.

[7] G. O. Matheson, L. Maffey-Ward, M. Mooney, K. Ladly, K. Fung and Y. Zhang, "Vibromyography as a quantitative measure muscle force production," Scand. J. Rehabil. Med., 29, pp.29-35, 1997.

[8] A. Torres, J.A. Fiz, J. Morera, A.E. Grassino, and R. Jané, "NonInvasive Measurement of Diaphragmatic Contraction Time in Dogs," 23th Ann. Conf. IEEE-EMBS, 2001.

[9] A. Torres, J.A. Fiz, J. Morera, A.E. Grassino,and R. Jané, “TimeFrequency representations of the diaphragmatic movement measured by a surface piezoelectric contact sensor in dogs," 25th Ann. Conf. IEEE-EMBS, 2003.

[10] A. Torres, J.A. Fiz, B. Galdiz, J. Gea, and R. Jané, "Non invasive assessment of respiratory muscle effort by means of the study of diaphragm movement registered with surface sensors. Animal model (dogs)," 26th Ann. Conf. IEEE-EMBS, 2004

[11] A. Torres, J.A. Fiz, B. Galdiz, J. Gea, J. Morera and R. Jané, "A Wavelet Multiscale Based Method to Separate the High and Low Frequency Components of Mechanomyographic Signals," 27th Ann. Conf. IEEE-EMBS, 2005

[12] Torres A., Fiz J.A., Galdiz B., Gea J., Morera J., Jané R., “Inspiratory Pressure Evaluation by means of the Entropy of Respiratory Mechanomyographic Signals," 28th Ann. Conf. IEEE-EMBS, pp: 5735-5738, 2006.

[13] Torres A., Fiz J.A., Jané R., Laciar E., Galdiz B., Gea J., Morera J., "Rényi Entropy and Lempel-Ziv Complexity of Mechanomyographic Recordings of Diaphragm Muscle as Indexes of respiratory Effort," 30th Ann. Conf. IEEE-EMBS, pp: 2112-2115, 2008.

[14] Sarlabous L., Torres A., Fiz J.A., Gea J., Galdiz J.B., Jané R., "Multistate Lempel-Ziv (MLZ) Index Interpretation as a Measure of Amplitude and Complexity Changes," 31th Ann. Conf. IEEE-EMBS, pp: 4375-4378, 2009.

[15] Sarlabous L., Torres A., Fiz J.A., Gea J., Martínez-Llorens J.M., Jané R., "Evaluation of the respiratory Muscular Function by means of Diaphragmatic Signals in COPD Patients," 31th Ann. Conf. IEEEEMBS, pp: 3925-3928, 2009. 